\title{
Fungal endophytes of some medicinal plant growing in northwestern coast of Egypt: Isolation, identification and two-way clustering analysis of its antimicrobial activity
}

Mohammad Magdy El-Metwally ( $\nabla$ mmmyco@gmail.com )

Damanhour Fac. of science https://orcid.org/0000-0002-3857-8794

Atia Mohamed Eisa

botany and microbiology, fac of science, damanhour university

Amal Al Mekawey

regional center of mycology and biotechnology, alazhar Univ.,

Samy F. Mahmoud

Taif University College of Science

Yasser El Halmouch

Damanhour University Faculty of Science

\section{Research Article}

Keywords: Fungal endophytes, Medicinal plants, Antimicrobial activity

Posted Date: August 23rd, 2021

DOl: https://doi.org/10.21203/rs.3.rs-828514/v1

License: (c) (1) This work is licensed under a Creative Commons Attribution 4.0 International License.

Read Full License 


\section{Abstract}

One of hidden mine of antibiotics is endophytic fungi especially that inhibited medicinal plants. In this regard, leaves, stems, fruits and bulbs of some commonly medicinal plants growing in Northwestern coast of Egypt were subjected for isolation of endophytic fungi with screening study of its antimicrobial activity. Practically, more than one hundred (101) endophytic fungal species isolated from Scorpiurus muricatus, Mellilotus indicus, Lotus polyphyllos, Ononis vaginalis, Nicotiana glauca, Lycium europaeum, Asphodelus aestivus, Echium angustifolium,Fagonia cretica, Pancratium maritimum,and Carduus getulus were tested in vitro for their antimicrobial activities against E. coli, Pseudomonas argenosa, Staphylococcus aureus, Bacillus subtilus, Candida albicanus, Candida glabrata, Penicillium expansum, Aspergillus flavus. The values of applied diversity indices revealed significant differences in presence, absence and abundance among endophytic fungal isolates. To the best of our knowledge, the present study is the first to report of Alternaria pluriseptata as endophytic species with most dominant and most active in its anitimicrobial activity among the isolated species. Seven distinctive groups were revealed from the two-way cluster analysis showing the intensity of antimicrobial activity against tested pathogens: Twenty-five percent of the isolates ( 26 strains assembled together in group $V$ ) exhibited no antimicrobial activity against all tested pathogens while six percent (6 isolates) assembled in group VII revealed high antimicrobial activity against five pathogens.

\section{Introduction}

The Northwestern coast of Egypt is one of the richest phyto-geographic regions with unique varieties of medicinal plant species. This coastal strip, with the weather being between pleasant and hot during summer and mild in winter and with its various ecosystems, offers important habitats for natural resources and their development. Hundred and eighty-eight plant species were recorded as medicinal species in the western Mediterranean habitats (Bidak et al.2013). Folk medicine was and still exploiting medicinal plants for thousands of years for their healing effects and health benefits. Isolation of plantderived drugs (very effective and have no side effects) is rapidly evolved in recent years. However, the natural resources of medicinal plants are challenging over-use practices at which the bioactive products accumulate at low levels in the native medicinal plants. Endomicrobiome of medicinal plant species may be responsible for the production of a wealth of explored and unexplored bioactive compounds (Venieraki et al 2017). Although, medicinal plants harbored many endophytic fungi that are active in the coproduction of active metabolites, a very few species were studied relative to their endophytic biology out of 420,000 plant species exist in nature. Endophytes contribute with considerable benefits to the host plant, where they act as a growth-promoting agent for host plants, besides, to enhance resistance to biotic and abiotic stresses (Hardoim et al.2015). Endophytic fungi grow inside plant tissues in specific ecosystems and produce multiples of secondary metabolites such as antioxidant, anticancer, immunomodulatory, antivirus, antituberculosis, anti-parasite and insecticides that show biological activities (Hussain et al.2014). In Egypt, screening medicinal plant species for endophytes and investigating their antimicrobial activities are a promising trend increasingly, attracted the 
microbiologist's attention lately. El-Maghraby et al. (2013) has explored three leguminous plant roots for Endophytic fungi in Egypt. Basheer et al. (2018) isolated the endophytic fungi from Avicennia marina and investigated the antibacterial and antifungal properties of their crude extracts against some important human pathogens. Plant growth-promoting activities for bacterial and fungal endophytes isolated from Teucrium polium L. were investigated by Saad El-Din Hassan (2017). Saad et al (2019) studied the potentiality of endophytic fungi as bio-control agents against the cotton leaf worm, Spodoptera littoralis. Although multiple of endophytic fungi studies were carried out on members of the Egyptian medicinal plants, Egyptian flora is full of extraordinary varieties waiting for exploring new good sources of biologically active compounds specifically from endophytic fungi. The present study aims at screening wider base of high value medicinal plants growing naturally in northwestern coast of Egypt (Matrouh area) and investigates the antimicrobial activities against some selective pathogens.

\section{Materials And Methods}

\section{Plant Collection and Identification}

Healthy (showing no visual disease) of eleven plant species belonging to seven families: Fabaceae (Scorpiurus muricatus L., Mellilotus indicus (L.) All., Lotus polyphyllos E. D. Clarke and Ononis vaginalis Vahl, Symb.) Solanaceae (Nicotiana glauca Graham and Lycium europaeum L.) Asphodelaceae (Asphodelus aestivus Brot.) Boraginaceae (Echium angustifolium Mill.) Zygophyllaceae (Fagonia cretica L.) Amaryllidaceae (Pancratium maritimum L.) and Asteraceae (Carduus getulus Pomel) were collected from Matrouh area at the west northern coast of Egypt. This phytogeographical region was selected according to the richness of habitats; sand dunes, rocky ridges, calcareous rocks and salt depressions that could play a pivotal role of existence and activity of the endophytes. Plant specimens have been identified to the species rank according to Boulos (2002).

\section{Surface Sterilization and Isolation of Endophytic Fungi}

Plant materials were washed under running tap water, followed by distilled water. Secondly, surface sterilization of plant materials were then done by sequentially rinsing with $70 \%$ ethanol for $30 \mathrm{sec}$., followed by $0.5 \%$ sodium hypochlorite for $2-3 \mathrm{~min}$, and then rinsing in $70 \%$ ethanol for nearly $2 \mathrm{~min}$, and finally with sterile distilled water 2-3 times. Plant materials were then dried in two folds of sterile filter papers. After sterilization, the plant materials were further cut (aseptically) to expose the interior surface to the nutrient media. For each plant, five segments from root stem and leaves were transferred to petri dishes containing potato dextrose agar (PDA) amended with chloramphenicol $500 \mathrm{mg} / \mathrm{l}$ (Al-Mahi et al.2003). The dishes were incubated at $28 \pm 2^{\circ} \mathrm{C}$ for $3-6$ days.

\section{Purification, Selection and Preservation of Endophytic Fungi}

Isolation from examined plants was done by the transfer of the hyphal tips to fresh PDA plates free of antibiotics to obtain pure cultures for identification. The purified endophytic fungal isolates were then transferred separately to PDA slants and maintained at $4^{\circ} \mathrm{C}$ till further use. 


\section{Identification of endophytic Fungi}

An Image analysis system, soft imaging system GmbH software (analySIS ® pro ver. 3.0) at The Regional Center for Mycology and Biotechnology Al- Azhar University (RCMB) was used for examining the alteration of the tested fungal morphological features. The cultures were examined microscopically after 5-7 days incubation for dermatophytes, 24 hours for yeast and/or yeast-like fungi and 3-4 days for other fungi, using light microscopy at magnification of X 400 for multicellular fungi, while $X 1000$ for unicellular fungi, using either phase-contrast or bright field optics under video camera. For each isolate 3-5 plates were prepared and a minimum of 20 microscopic fields were examined. Raper and Thom C (1949), Gilman (1957), Raper and Fennel (1965), Ellis and Booth (1971) and Domsch et al. (1980).

\section{Fermentation}

Twenty five $\mathrm{ml}$ of malt glucose broth was distributed in $100 \mathrm{ml}$ conical flasks and autoclaved at $121^{\circ} \mathrm{C}$ for $15 \mathrm{~min}$. The isolated endophytic fungal strains were inoculated aseptically into all flasks. The flasks were incubated at $28 \pm 2^{\circ} \mathrm{C}$ for 10 days for growth. The flasks were examined periodically for any contamination. After 10 days, culture media were centrifuged at $6,000 \mathrm{rpm}$ for $35 \mathrm{~min}$. Antimicrobial activity of culture supernatants of endophytes was evaluated by agar well diffusion method using Mueller Hinton agar medium for the bacteria and Sabouraud Dextrose agar for yeast and fungi. By using sterile cork borer, about $5 \mathrm{~mm}$ size wells were made and $200 \mu \mathrm{l}$ of each culture supernatant was added into it. The diameter of inhibition zone (millimeter) were measured after incubation at $37 \pm 2^{\circ} \mathrm{C}$ for $24 \mathrm{~h}$ for bacteria and at $28 \pm 2^{\circ} \mathrm{C}$ for 3 days for fungi (Sutjaritvorakul et al.2011).

\section{Determination of Antimicrobial activity}

List of microbial pathogens included E.coli, Staphylococcus aureus, Bacillus subtilus, Pseudomonas argenosa, C. albicanus, Penicillium expansum, Candida glabrata, Aspergillus flavus (All microorganisms were maintained at $4^{\circ} \mathrm{C}$ on nutrient agar slants for bacteria, Malt Glucose Agar slants for fungi.) Agar Disc well diffusion method was used for the antimicrobial susceptibility testing. Antimicrobial potentialities were expressed as the diameter of inhibition zones. Extracts were examined as antimicrobial agent against all microbial isolates. Inoculum suspensions of all bacteria and fungi isolates were spread on the surface media. Four equidistant $(1 \mathrm{~cm}$ diameter) holes were made in the agar using sterile cork borer in media plates $(10 \times 10 \mathrm{~cm})$, which had previously been seeded with bacteria and/or fungi tested, were filled by $100 \mu \mathrm{L}$ of each extract. Discs injected with $100 \mu \mathrm{L}$ of respective $50 \mathrm{mM}$ sodium phosphate buffer and organic solvent were used as negative control. Standard antibiotic was used as positive control ciprofloxacin (100 $\mu \mathrm{L} /$ disc) (Oxoid) and fluconazole $(100 \mu \mathrm{L} /$ disc) (Oxoid) were used as a positive reference for bacteria and fungi, respectively. Plates were left in a cooled incubator at 4 $( \pm 2)^{\circ} \mathrm{C}$ for one hour and then incubated at $37( \pm 2)^{\circ} \mathrm{C}$ for 24 hour for bacterial growth and $28^{\circ} \mathrm{C}$ for 48 hours for fungal growth. Inhibition zones developed due to active extract ingredients were measured after 24-48 hours of incubation. The experiments were carried out in triplicates and

the inhibition zone was measured with the help of standard scale (Norrel \& Messley, 1997) 


\section{Data Analysis}

Data analysis was accomplished using PC-ORD ver. 5 to perform Two Way Cluster analysis, it is an agglomerative clustering method in which data matrix is classified twice, one time for the rows and the other is for the columns. Clustering dendrogram performed based on Sorensen and Flexible beta of -0.25 . Fungal endophytic Species frequency and Diversity indices

Frequency values of the final list of identified isolates were calculated based on the number of times a particular species presents in each plant organ, relative frequency values were calculated by dividing the frequency value for a particular species by the values for all species and multiply by $100 \%$. The isolation rate (IR, \%) was calculated by dividing the total number of isolates from specific tissues by the overall isolates number.

Species richness (Alpha diversity) was calculated after Whittaker (1972), who described it as the diversity within a particular area or ecosystem and it is expressed by the number of species in each stand.

\section{Shannon Index:}

$$
\mathbf{H}^{\prime}=-\sum_{i=\mathbf{1}}^{S} \boldsymbol{P}_{i} \ln \boldsymbol{P}_{i},
$$

Where pi is proportion of S made up of the ith species

$$
D=1-\left(\frac{\sum n(n-1)}{N(N-1)}\right)
$$

Where $\mathrm{n}=$ the total number of organisms of a particular species and $\mathrm{N}=$ the total number of organisms of all species

The species evenness (E): calculated after Pielou (1966) by the following formula: $E=H / \operatorname{Ln}(S)$

Where $\mathrm{H}$ is Shannon value and $(\mathrm{S})$ is the richness for each organ. Evenness defined as a diversity index, a measure of biodiversity which quantity how equal the community is numerically.

\section{Results}

\section{Isolation and identification of endophytic fungi}

A total of 101 endophytic fungal isolates belonging to 18 genera and 35 fungal species were isolated from vegetative organs of 11 selected medicinal plants growing naturally on the north-western coast of Egypt (Table 1). The isolated genera namely, Alternaria, Phoma, Mucor, Scytalidium, Pythium, Ulocladium, Spegazzinia, Sarcinomyces Aspergillus, Cladosporium, Penicillium, Clafosporium, Rhizopus, Fusarium, Nigrosora, Trichocladium, Leptosphaeria and Aureobasidium. 
Table 1

List of endophytic fungal species isolated from 11 selected medicinal plants growing naturally on the north-western coast of Egypt

\begin{tabular}{|c|c|c|c|}
\hline Name of plants & $\begin{array}{l}\text { Plant } \\
\text { organs }\end{array}$ & $\begin{array}{l}\text { Fungal code } \\
\text { No. }\end{array}$ & Name of fungal isolates \\
\hline \multirow[t]{6}{*}{ A- Scorpiurus muricatus L. } & Fruits & 1 & Alternaria pluriseptata \\
\hline & \multirow[t]{2}{*}{ Stem } & 2 & Phoma dennissii \\
\hline & & 3 & Alternaria pluriseptata \\
\hline & \multirow[t]{3}{*}{ Leaves } & 4,5 & Alternaria phragmospora \\
\hline & & 6 & Alternaria alternaria \\
\hline & & 7 & Alternaria pluriseptata \\
\hline \multirow[t]{9}{*}{ B- Faganiu cretica L. } & \multirow[t]{5}{*}{ Leaves } & 8 & Alternaria pluriseptata \\
\hline & & 9 & Phoma glomerata \\
\hline & & 10 & Scytalidium japonicum \\
\hline & & 11 & Pythium nayoroense \\
\hline & & 16 & Ulocladium atrum \\
\hline & \multirow[t]{2}{*}{ Stem } & 12 & Alternaria pluriseptata \\
\hline & & 13 & Alternaria chlamydospora \\
\hline & \multirow[t]{2}{*}{ Fruits } & 14 & Mucor hiemalis \\
\hline & & 15 & Alternaria pluriseptata \\
\hline \multirow[t]{8}{*}{ C- Mellilotus indicus (L.)All. } & \multirow[t]{6}{*}{ Leaves } & 17 & Penicillium aurantiogriseum \\
\hline & & 18 & Clafosporium cladosporioides \\
\hline & & 19 & Rhizopus stolonifer \\
\hline & & 20 & Sarcinomyces phaeomriformis \\
\hline & & 21 & Alternaria pluriseptata \\
\hline & & 22 & Aspergillus niger \\
\hline & \multirow[t]{2}{*}{ Stem } & 23 & Penicillium aurantiogriseum \\
\hline & & 24 & Penicillium marneffei \\
\hline
\end{tabular}




\begin{tabular}{|c|c|c|c|}
\hline Name of plants & $\begin{array}{l}\text { Plant } \\
\text { organs }\end{array}$ & $\begin{array}{l}\text { Fungal code } \\
\text { No. }\end{array}$ & Name of fungal isolates \\
\hline & Fruits & 25 & Alternaria pluriseptata \\
\hline & & 26 & Alternaria phragmospora \\
\hline & & 27 & Spegazzinia pakeri \\
\hline & & 28 & Sarcinomyces phaeomriformis \\
\hline & & 29 & Cladosporium tenuissium \\
\hline & & 30 & Penicillium expansum \\
\hline & & 31 & Penicillium roqueforti \\
\hline \multirow[t]{9}{*}{ D- Carduus getulus Pomel } & Stem & 32 & Alternaria pluriseptata \\
\hline & & 33 & Ulocladium alternariae \\
\hline & & 34 & Rhizopus oryzae \\
\hline & Leaves & 35 & Alternaria pluriseptata \\
\hline & & 36 & Rhizopus microspores \\
\hline & Fruits & 37 & Pythium elongutum \\
\hline & & 38 & Pythium nayoroerse \\
\hline & & 39 & Alternaria pluriseptata \\
\hline & & 40 & Pencillium expansum \\
\hline \multirow[t]{8}{*}{ E- Pancratium maritimum L. } & Pulp & 41 & Mucor hiemalis \\
\hline & & 42 & Aspergillus niger \\
\hline & Leaves & 43 & Alternaria pluriseptata \\
\hline & & 44 & Alternaria alternaria \\
\hline & & 45 & Pythium nayoroerse \\
\hline & & 46 & Cladosporium nigrellam \\
\hline & & 47 & Cladosporium cladosporioides \\
\hline & & 48 & Penicillium marneffei \\
\hline
\end{tabular}




\begin{tabular}{|c|c|c|c|}
\hline Name of plants & $\begin{array}{l}\text { Plant } \\
\text { organs }\end{array}$ & $\begin{array}{l}\text { Fungal code } \\
\text { No. }\end{array}$ & Name of fungal isolates \\
\hline \multirow[t]{12}{*}{ F- Asphodelus aestivus Brot. } & \multirow[t]{6}{*}{ Leaves } & 49 & Fusarium verticilloioides \\
\hline & & 50 & Cladosporium nigrellam \\
\hline & & 51 & Rhizopus stolonifer \\
\hline & & 52 & Alternaria pluriseptata \\
\hline & & 53 & Ulocladium alternariae \\
\hline & & 54 & Fusarium oxysporum \\
\hline & \multirow[t]{6}{*}{ Stem } & 55 & Fusarium verticilloioides \\
\hline & & 56 & Fusarium oxysporum \\
\hline & & 57 & Alternaria pluriseptata \\
\hline & & 58 & Cladosporium nigrellam \\
\hline & & 59 & Rhizopus stolonifer \\
\hline & & 60 & Penicillium raistrickii \\
\hline \multirow{5}{*}{$\begin{array}{l}\text { G- Lotus polyphyllos E. D. } \\
\text { Clarke }\end{array}$} & \multirow[t]{2}{*}{ Stem } & 61 & Alternaria pluriseptata \\
\hline & & 62,63 & Ulocladium alternariae \\
\hline & \multirow[t]{3}{*}{ Leaves } & 64 & Ulocladium alternariae \\
\hline & & 65 & Alternaria pluriseptata \\
\hline & & 66,67 & Aspergillus niger \\
\hline \multirow[t]{10}{*}{ H- Lycium europaeum L. } & \multirow[t]{10}{*}{ Leaves } & 68 & Rhizopus stolonifer \\
\hline & & 69 & Trichocladium asperum \\
\hline & & 70 & Pythium nayoroerse \\
\hline & & 71 & Nigrosora sphaeria \\
\hline & & 72 & Mucor hiemalis \\
\hline & & 73 & Rhizopus stolonifer \\
\hline & & 74 & Penicillium marneffei \\
\hline & & 75 & Ulocladium atrum \\
\hline & & 76 & Alternaria chlamydospora \\
\hline & & 77 & Aspergillus niger \\
\hline
\end{tabular}




\begin{tabular}{|c|c|c|c|}
\hline Name of plants & $\begin{array}{l}\text { Plant } \\
\text { organs }\end{array}$ & $\begin{array}{l}\text { Fungal code } \\
\text { No. }\end{array}$ & Name of fungal isolates \\
\hline & \multirow[t]{5}{*}{ Stem } & 78 & Alternaria alternata \\
\hline & & 79 & Rhizopus stolonifer \\
\hline & & 80 & Alternaria pluriseptata \\
\hline & & 81 & Nigrosora sphaeria \\
\hline & & 82 & Ulocladium atrum \\
\hline \multirow[t]{5}{*}{ J- Echium angustifolium Mill. } & \multirow[t]{5}{*}{ Leaves } & 83 & Aspergillus terrus \\
\hline & & 84 & Coccidioides immitis \\
\hline & & 85 & Rhizopus stolonifer \\
\hline & & 86 & Alternaria pluriseptata \\
\hline & & 87 & Spegazzinia pakeri \\
\hline \multirow[t]{10}{*}{ K- Nicotiana glauca Graham } & \multirow[t]{5}{*}{ Leaves } & 88 & Cladosporium nigrellam \\
\hline & & 89 & Rhizopus stolonifer \\
\hline & & 90 & $\begin{array}{l}\text { Cladosporium } \\
\text { sphaerospermum }\end{array}$ \\
\hline & & 91 & Alternaria pluriseptata \\
\hline & & 92 & Spegazzinia pakeri \\
\hline & \multirow[t]{5}{*}{ Stem } & 93 & Alternaria chlamydospora \\
\hline & & 94 & Alternaria pluriseptata \\
\hline & & 95 & Ulocladium atrum \\
\hline & & 96 & Leptosphaeria senegalensis \\
\hline & & 97 & Aureobasidium pellalanus \\
\hline \multirow{4}{*}{$\begin{array}{l}\text { L- Ononis vaginalis Vahl, } \\
\text { Symb. }\end{array}$} & \multirow[t]{4}{*}{ Leaves } & 98 & Alternaria pluriseptata \\
\hline & & 99 & Ulocladium atrum \\
\hline & & 100 & Aspergillus niger \\
\hline & & 101 & Cladosporium nigrellam \\
\hline
\end{tabular}

\section{Endophytes frequency and diversity indices}

A list of 101 isolates and their frequencies, relative frequencies were recorded in the investigated plant tissues. The detailed results summarized in Table 2 , revealed that $56 \%$ isolates were obtained from plant leaf tissues, $27 \%$ from the stem, $15 \%$ from fruits and $2 \%$ from pulps. The dominant endophytic species 
recorded with high relative frequencies were Alternaria pluriseptata (20\%) with 14 active isolates against the tested pathogens, Rhizopus stolonifer (8\%) four of them were active, Aspergillus niger (6\%), four of them were active, Ulocladium atrum, Cladosporium nigrellam, and Ulocladium alternariae share the same relative frequency (5\%) and gave positive results for two, one and two as active strains respectively and Pythium nayoroense (4\%) with $50 \%$ active isolates. The rest of the listed endophytic fungi were recorded with lower relative frequencies. 
Table 2

Isolated fungi, frequency $(F)$, relative frequency $(R F)$, isolation rate (IR \%), Richness (S), Species diversity, Shannon index $(H)$, Simpson diversity (1-D) and Evenness values (E) tissues.

\begin{tabular}{|c|c|c|c|c|c|c|c|c|}
\hline \multirow[t]{2}{*}{ NO } & \multirow[t]{2}{*}{ Endophyte species } & \multirow[t]{2}{*}{ (F) } & \multirow{2}{*}{$\begin{array}{l}\text { R.F } \\
\text { (\%) }\end{array}$} & \multicolumn{4}{|c|}{ organs } & \multirow{2}{*}{$\begin{array}{l}\text { No of } \\
\text { active } \\
\text { isolates }\end{array}$} \\
\hline & & & & Fruit & Leave & stem & pulp & \\
\hline 1 & Alternaria pluriseptata & 21 & 20 & 4 & 10 & 7 & 0 & 14 \\
\hline 2 & Phoma dennissii & 1 & 1 & 0 & 0 & 1 & 0 & 1 \\
\hline 3 & Alternaria phragmospora & 3 & 3 & 1 & 2 & 0 & 0 & 3 \\
\hline 4 & Alternaria alternaria & 3 & 3 & 0 & 2 & 1 & 0 & 2 \\
\hline 5 & Phoma glomerata & 1 & 1 & 0 & 1 & 0 & 0 & 1 \\
\hline 6 & Scytalidium japonicum & 1 & 1 & 0 & 1 & 0 & 0 & 1 \\
\hline 7 & Pythium nayoroense & 4 & 4 & 1 & 3 & 0 & 0 & 2 \\
\hline 8 & Ulocladium atrum & 5 & 5 & 0 & 3 & 2 & 0 & 2 \\
\hline 9 & Alternaria chlamydospora & 3 & 3 & 0 & 1 & 2 & 0 & 2 \\
\hline 10 & Mucor hiemalis & 3 & 3 & 1 & 1 & 0 & 1 & 1 \\
\hline 11 & $\begin{array}{l}\text { Penicillium } \\
\text { aurantiogriseum }\end{array}$ & 2 & 2 & 0 & 1 & 1 & 0 & 1 \\
\hline 12 & $\begin{array}{l}\text { Cladosporium } \\
\text { cladosporioides }\end{array}$ & 2 & 2 & 0 & 2 & 0 & 0 & 1 \\
\hline 13 & Rhizopus stolonifer & 8 & 8 & 0 & 6 & 2 & 0 & 4 \\
\hline 14 & $\begin{array}{l}\text { Sarcinomyces } \\
\text { phaeomriformis }\end{array}$ & 2 & 2 & 1 & 1 & 0 & 0 & 2 \\
\hline 15 & Aspergillus niger & 6 & 6 & 0 & 5 & 0 & 1 & 4 \\
\hline 16 & Penicillium marneffei & 3 & 3 & 0 & 2 & 1 & 0 & 3 \\
\hline 17 & Spegazzinia pakeri & 3 & 3 & 1 & 2 & 0 & 0 & 3 \\
\hline 18 & Cladosporium tenuissium & 1 & 1 & 1 & 0 & 0 & 0 & 0 \\
\hline 19 & Ulocladium alternariae & 5 & 5 & 0 & 2 & 3 & 0 & 1 \\
\hline 20 & Rhizopus oryzae & 1 & 1 & 0 & 0 & 1 & 0 & 1 \\
\hline 21 & Rhizopus microspores & 1 & 1 & 0 & 1 & 0 & 0 & 0 \\
\hline 22 & Pythium elongutum & 1 & 1 & 1 & 0 & 0 & 0 & 1 \\
\hline 23 & Cladosporium nigrellam & 5 & 5 & 0 & 4 & 1 & 0 & 2 \\
\hline
\end{tabular}




\begin{tabular}{|c|c|c|c|c|c|c|c|c|}
\hline \multirow[t]{2}{*}{ NO } & \multirow[t]{2}{*}{ Endophyte species } & \multirow[t]{2}{*}{ (F) } & \multirow{2}{*}{$\begin{array}{l}\text { R.F } \\
\text { (\%) }\end{array}$} & \multicolumn{4}{|c|}{ organs } & \multirow{2}{*}{$\begin{array}{l}\text { No of } \\
\text { active } \\
\text { isolates }\end{array}$} \\
\hline & & & & Fruit & Leave & stem & pulp & \\
\hline 24 & Fusarium verticilloioides & 2 & 2 & 1 & 1 & 0 & 0 & 2 \\
\hline 25 & Fusarium oxysporum & 2 & 2 & 0 & 1 & 1 & 0 & 0 \\
\hline 26 & Penicillium raistrickii & 1 & 1 & 0 & 0 & 1 & 0 & 1 \\
\hline 27 & Trichocladium asperum & 1 & 1 & 0 & 1 & 0 & 0 & 0 \\
\hline 28 & Nigrosora sphaeria & 2 & 2 & 0 & 1 & 1 & 0 & 1 \\
\hline 29 & Aspergillus terrus & 1 & 1 & 0 & 1 & 0 & 0 & 1 \\
\hline 30 & Coccidioides immitis & 1 & 1 & 0 & 1 & 0 & 0 & 1 \\
\hline 31 & $\begin{array}{l}\text { Cladosporium } \\
\text { sphaerospermum }\end{array}$ & 1 & 1 & 0 & 1 & 0 & 0 & 0 \\
\hline 32 & $\begin{array}{l}\text { Leptosphaeria } \\
\text { senegalensis }\end{array}$ & 1 & 1 & 0 & 0 & 1 & 0 & 1 \\
\hline 33 & Aureobasidium pellalanus & 1 & 1 & 0 & 0 & 1 & 0 & 1 \\
\hline 34 & Penicillium expansum & 2 & 2 & 2 & 0 & 0 & 0 & 0 \\
\hline \multirow[t]{4}{*}{35} & Penicillium roqueforti & 1 & 1 & 1 & 0 & 0 & 0 & 0 \\
\hline & Total no of isolates & 101 & 100 & 15 & 57 & 27 & 2 & 75 \\
\hline & Isolation rate (\%) & & & 15 & 56 & 27 & 2 & \\
\hline & Species richness (S) & & & 11 & 26 & 16 & 2 & \\
\hline \multirow[t]{3}{*}{$\begin{array}{l}\text { Species } \\
\text { diversity }\end{array}$} & $\begin{array}{l}\text { Simpson's index of } \\
\text { diversity }(1-D)\end{array}$ & & & 0.93 & 0.95 & 0.92 & 1 & \\
\hline & Shannon index $(\mathrm{H})$ & & & 2.14 & 2.55 & 2.59 & 0.7 & \\
\hline & Evenness (E) & & & 0.89 & 0.78 & 0.93 & 1 & \\
\hline
\end{tabular}

On the other hand, the richness and the diversity of the isolated endophytic fungi were calculated; plant leaf tissues settled the highest richness value with 26 species followed by stem ( 16 species) and 11 species for fruits. Simpson diversity indices values were, 0.95 for leaf tissues, 0.93 for fruits and 0.92 for stem, consistently; Shannon index values $(H=2.55$ in leaf tissues), $(H=2.59$ in stem tissues $)$ and $(H=$ 2.14 in fruits) while recorded in pulps as the lowest value $(H=0.7)$. Evenness indice4s for the leaf $(E=$ $0.78)$, for the stem $(E=0.93)$ and for fruits $(E=0.89)$

\section{Antimicrobial activity of isolated endophytic fungi}


Based on the similarity measure between the different endophytes activity, the antimicrobial activity data against eight selected significant pathogens were subjected to two-way clustering analysis. The resulted dendrogram revealed seven contiguous groups of fungi: The superimposed groups are discriminated at high resolution at the cut level of 0.65 . Hierarchically, the resulted groups are arranged on the figure (1) and the pattern of each assemblage is clarifying as following:

\section{Group I}

this group separated firstly at the base of the tree with 14 isolates and showed high activity range against E. coli (mean $23 \mathrm{~mm}$ clear zone), Table 3. This group divided into three subgroups. The first subgroup; Alternaria pluriseptata (35), Alternaria phragmospora(5), Rhizopus stolonifer(51 and 68), Penicillium marneffei (48), Aspergillus niger (77), Cladosporium nigrellam (88), and Ulocladium alternariae (53) isolated from leaf tissues and revealed high activity only against $E$. coli. Cladosporium cladosporioides (18), Penicillium aurantiogriseum (23), Alternaria phragmospora (26) isolated from Mellilotus indicus and inhibited Aspergillus flavus, E.coli, and Pseudomonas argenosa were included in the second subgroup.

The third subgroup represented by Scytalidium japonicum (10), Cladosporium cladosporioides (47), and Alternaria pluriseptata (98), isolated from leaf tissues and showed varied activity against $E$. coli,

Pseudomonas argenosa, Bacills subtilus, Aspergillus flavus, and Penicillium expansum.

Table 3

Two-way cluster groups (I - VII), No. of isolated endophytes in each group, inhibition zone $(\mathrm{main} / \mathrm{mm})$ and the most pathogens affected by groups

\begin{tabular}{|llll|}
\hline Groups & No of & inhibition & Most affected pathogens \\
isolates & $\begin{array}{l}\text { zone } \\
\text { main } \\
(\mathrm{mm})\end{array}$ & \\
\hline I & 14 & 23 & E. Coli \\
\hline II & 10 & 20 & Staphylococcus aureus \\
\hline III & 10 & 13 & Candida albicans \\
\hline IV & 15 & 17 & Pseudomonas argenosa \\
\hline V & 28 & 0 & Not active \\
\hline VI & 18 & 21 & Bacillus subtilus \\
\hline VII & 6 & 20 & $\begin{array}{l}\text { Bacillus subtilus, Staphylococcus aureus, Penicillium expansu, } \\
\text { Aspergillus flavus, Pseudomonas argenosa and E.coli }\end{array}$ \\
\hline
\end{tabular}

\section{Group II}

Ten fungal strains assembled in group II, inhibited mainly and strongly Staphylococcus aureus with mean inhibition zone of $20 \mathrm{~mm}$. Table 3. Mucor hiemalis (14), Ulocladium alternariae (63), Pythium nayoroense 
(38), Alternaria pluriseptata (43 and 21), Fusarium oxysporum (56) included in active subgroup against only Staphylococcus aureus. Fusarium verticilloioides (55) appeared in the same group but showed activity against Candida albicanus in addition to its activity against Staphylococcus aureus. The second subgroup represented by two isolates of Alternaria pluriseptata (12 and 15) and one isolate of Sarcinomyces phaeomriformis (20) was multiactive strains against E. coli, Staphylococcus aureus and Candida albicans.

\section{Group III}

Ten strains represented this group; seven isolate from leaf tissues, two other from stem and one from fruit tissues. Three different patterns of activity for these 10 endophytes with moderate inhibitory zones (main = $13 \mathrm{~mm}$ ). Alternaria pluriseptata (8), Alternaria chlamydospora (13), Aspergillus niger (22), Rhizopus stolonifer (85) were similar in their activity against Pseudomonas argenosa, Staphylococcus aureus and Aspergillus flavus. While Fusarium verticilloioides (49) and Alternaria pluriseptata (61) isolated from Asphodelus aestivus and Lotus polyphyllo respectively showed high activity against Pseudomonas argenosa, Staphylococcus aureus, Aspergillus flavus and Candida albicans in a different pattern. Cladosporium nigrellam(58), Alternaria alternaria (44), Phoma glomerata (9), and Spegazzinia pakeri (27) isolated from leaf tissues of Asphodelus aestivus, Pancratium maritimum, Fagonia cretica and Mellilotus indicus respectively, mostly inhibited Candida albicans and Aspergillus flavus in third pattern.

\section{Group IV}

Fungal isolates in group IV characterized by distinct antimicrobial activity against Pseudomonas argenosa with main inhibition zone of $17 \mathrm{~mm}$. table (3). Fifteen strains were incorporated into this group (graph 1) mostly isolated from investigated leaf tissues and assembled in two subgroups. Rhizopus stolonifer (19), Penicillium raistrickii (60), Aspergillus niger (66), Nigrosora sphaeria (71), Rhizopus oryzae (34), Spegazzinia pakeri (87), Ulocladium atrum (82), Pythium elongutum (37), Alternaria pluriseptata (57), Aspergillus niger and Coccidioides immitis (84) aggregated in a subset and behaved in a similar manner against one pathogen, Pseudomonas argenosa. The other subgroup, Alternaria phragmospora (26), Ulocladium atrum (75), Penicillium expansum (30) and Penicillium marneffei revealed high antimicrobial activity against two pathogens, Candida glabrata and Pseudomonas argenosa.

\section{Group V (Inactive group)}

Twenty-six strains assembled together in group $V$ that gave negative effect against all tested pathogens. Plant species, six inactive strains obtained from Lycium europaeum, four isolates from Carduus getulus, and three inactive strains from both Asphodelus aestivus and Nicotiana glauca. Other plant species represented by at least one inactive strain. Alternaria phragmospora (4) and Alternaria pluriseptata (76) grouped as closely related to group V However these two strains aere active against Candida glabrata and Penicillium expansum, respectively graph (1). 


\section{Group VI}

This group contained 18 strains isolated from all plant species, shared the ability of inhibiting Bacillus subtilis with inhibition zone (main $=21 \mathrm{~mm}$ ) table (3). and differentiated from each other in terms of antimicrobial activity against other tested pathogens. Group VI divided into smaller six subsets based on the number of pathogens affected by its endophytic fungi. Four isolates, Aspergillus niger (100), Sarcinomyces phaeomriformis (28), Rhizopus stolonifer (59) Cladosporium nigrellam (101) were active against four different pathogens. While, Rhizopus stolonifer (73), Nigrosora sphaeria (81), Pythium nayoroense (45) revealed Antimicrobial activity against three pathogens. Phoma dennissii (2), Cladosporium nigrellam (46), Ulocladium atrum (99) and Alternaria pluriseptata (65) gave positive effect against one pathogen while the rest of the isolates in group VI activated positively against two pathogens.

\section{Group VII (Multi-active group)}

six isolates classified into two subgroups identified multivariate responses against all pathogens with wide inhibition zones (main $=20 \mathrm{~mm}$ ). Two strains of Alternaria pluriseptata (6 and 7) isolated from Scorpiurus muricatu leaf tissues and one strain of Rhizopus stolonifer isolated from the leaf tissues of Nicotiana glauca revealed high antimicrobial activity against all tested pathogens except for $C$. albicanus. On the other hand; Penicillium marneffei (24) isolated from Mellilotus indicus, Alternaria pluriseptata (32) isolated from Carduus getulus and Spegazzinia pakeri (92) isolated from Nicotiana glauca revealed strong activity against all tested pathogens except $E$. coli.

\section{Discussion}

Endophytes are a big hidden world of unique fungal isolates which have different biological activities and produce bioactive compounds with a high level of structural diversity. It can be considered as major reservoirs of natural bioactive metabolites which are very efficient in industrial, agricultural and medicinal applications (Selim et al. 2016). In the present work, the selection of medicinal plants in Northwestern coast of Egypt based on the little previous work on isolation and identification of endophytes from these plants growing in this area. Our isolation strategy was based on each part of the plant because the endophytes isolates depend not only on the plant, but also on the tissue of the plant (Golinska et al. 2015). From 11 medicinal plant species 101 fungal isolates belonging to 18 genera and 35 fungal species were isolated and the most frequent endophytic fungal species is Alternaria pluriseptata, To discuss these results, no isolation or identification trails have been done in fungal endophytes of these plants of these area, but in comparison with other published studies these findings is more diverse than that recorded By Hassanein et al. (2016) who isolated Thirty endophytic fungal species from green onion, basil, green pepper, roselle, mint, watercress, tagetes and white radish and more also than that isolated by Ebrahimia et al. (2010) who isolated only 8 endophytic fungi from random leaves and branches samples of five native medicinal plants in iran namely, Stachys lavandulifolia, Rumex pulcher, Hypericum scabrum, Starja bachteriarica and Achillea kellalensis. And more than that recorded by Raviraja (2005) who 
isolated eighteen species of endophytic fungi from bark, stem and leaf segments of five medicinal plant species growing within the Kudremukh range in the Western Ghats of India. In the other hand Son (2002) obtained 121 fungal isolates from 62 different types of medicinal plants.

The present study declares that fungal endophytes showed significant differences in their presence, absence and abundance in the study plants. These results were consistent with the previous reports (Hassanein et al. 2016). In addition the endophytic fungi have been found in different tissues of a single plant which is a tissue specificity reflection and might be also due to other factors like, degree of exposure to sun, air, rain, wind, moisture and aeration conditions and nutrients availability (Andreote et al. 2014), this explain that endophytic fungal species and their population structure of even the same host plant species from different regions normally presented very low similarity degree (Jiang et al.2010).

The variation in number and type of isolated fungal endophytes can be explained by some medicinal plants harbored more endophytic fungi than others and some of the common endophytes not only existed in more plant hosts but also had higher relative frequencies within each of the hosts. In contrast, some other endophytic fungi were detected in only one given plant host (Huang et al. 2008) moreover some studies have shown that endophytes colonization can be stimulated by host plant species, geographic location, seasonality and different tissues of the same plant (Yao et al. 2017).

In our study the most dominant and most active in its microbial activity is Alternaria pluriseptata, to the pest of our knowledge this is the first report about this species as endophytes. Generally alternaria genus is one of active and common endophytes, Tonial et al. (2016) reported it on Schinus terebinthifolius endophytes collected in an unspecified location in South America. The endophytic isolates were identified as Alternaria spp. Sect. Alternate and from 11 different medicinal plants collected from Tamil Nadu, India, the most frequently isolated fungi were Alternaria sp. (Palanichamy et al.2018) parallel to this study Mani et al. (2015) isolated Alternaria citrimacularis and Alternaria alternate from the medicinal tree Aegle marmelos, collected in India. Most of the Alternaria sp produces many of biologically active secondary metabolites such as Hexahydroaltersetin, Altersetin, Dihydroaltersetin, Equisetin,, Zinnimide, Deprenylzinnimide, DesmethyldestruxinB, Cichorine, HomodestruxinB, Alternariol, Alternariol 5-0-sulfate, and Macrosporin (Lou et al.2013). Elgorban et al (2019) isolated Thirty-seven bioactive chemical compounds were recorded in the crude extracts of Alternaria sp. (A8) using GC-MS Thirteen major bioactive compounds were recorded namely: 1,2-Benzenedicarboxylic acid, bis (2-ethyhexyl) ester representing $51.15 \%$ of the crude extract, 6,8-dimethoxy-4-methyl-4H- chromene (10.87), 2,5Cyclohexadien-1-one, 2,6-bis (1,1-dimethylethyl)-4-ethylidene- (3.94\%), Cetene (3.32\%), 1,2Benzenedicarboxylic acid, dibutyl ester (3.23\%), 1-Octadecene (2.64\%), Benzene ethanol (2.47\%), 1Octdecene (2.21\%), Cycloe- icosane (1.96\%), 1-Butanol, 3- methyl-, acetate sopentyl alcohol, acetate (1.91\%), 1-Tetradecene (1.77\%), Naphthalene (1.69\%), Phenol, 2,4-di-t-butyl-6-nitro (1.59\%). These compounds showed strong antibacterial activity in combination Johann et al. (2012) recorded the biphenyl derivative compound altenusin, produced by the endophytes Alternaria $s p$. This compound is 50fold more active against Paracoccidioides brasiliensis than the reference trimethroprim/sulfamethoxazole compounds. It was suggested that altenusin possibly affects cell wall 
synthesis or assembly (Johann et al.2012). In 2009, Gu (2009) reported on the compounds altechromone A and herbarin A produced by Alternaria brassicicola ML-P08, an endophyte of Chinese Malus halliana. Altechromone A showed activity against B. subtilis, E. coli, C. albicans and Pseudomonas fluorescens Herbarin A showed activity against $C$. albicans and Trichophyton rubrum. The habitats of the Egyptian flora possess a large number of potential aromatic and medicinal plant species that accumulate and concentrate secondary metabolites as response of the environmental stresses.

The present study shed the light on the promising sources of the Egyptian medicinal plant regarding secondary metabolic contents. The habitats of the Egyptian flora possess a large number of potential aromatic and medicinal plant species that accumulate and concentrate secondary metabolites as response of the environmental stress. In conclusion, this study showed the diversity of species and activity of isolated fungi. Which opens endless horizons to more and more research in medicinal plants endophytes.

\section{Declarations}

\section{CONFLICT OF INTERESTS}

The authors declare that there is no conflict of interests.

\section{ACKNOWLEDGMENTS}

We appreciate and thank Taif University for the financial support for Taif University Researchers Supporting Project (TURSP-2020/138), Taif University, Taif, Saudi Arabia.

\section{References}

1. Al-Mahi I, letidal A, Eihab I (2013) Antibacterial activity of endophytic fungi extracts from the medicinal plant Kigelia africana. Egypt Acad J Biolog Sci 5:11-19

2. Andreote FD, Gumiere T, Durrer A (2014) Exploring interactions of plant microbiomes. Sci Agric 71:528-539

3. Basheer M, Mekawy A, El Kafrawy S, Abouzeid M (2018) Antimicrobial activities of endophytic fungi of red sea aquatic plant Avicennia marina. Egypt J Microbiol 53:231-240

4. Bidak LM, Heneidy SZ, Shaltout KH, Al-Soudany Y (2013) Current status of the wild medicinal plants in the western Mediterranean coastal region, Egypt. J Ethnobiol Trad Med Photon 120:566-584

5. Booth C. The genus Fusarium (1971) Common wealth Mycological Institute, Kew, Surrey, 237

6. Boulos L. Flora of Egypt. (Azollaceae-Oxalidaceae) (1999) A1-Hadara Publishing, Cairo, Egypt

7. Boulos L. Flora of Egypt. (Geraniaceae-Boraginaceae) (2000) A1-Hadara Publishing, Cairo, Egypt

8. Boulos L. Flora of Egypt. (Verbinaceae-Compositae) (2002) AlHadara Publish, Cairo, Egypt 
9. Domsch KH, Gams W, Anderson TH (1980) Compendium of soil fungi. Academic Press Inc., New York

10. Ebrahimia A, Asghariana S, Habibianb S (2010) Antimicrobial activities of isolated endophytes from some Iranian native medicinal plants Iran. J Pharm Sci Summer 6:3217-3222

11. Elgorban A, Bahkali A, Al Farraja D, Abdel-Wahab M (2019) Natural products of Alternaria sp., an endophytic fungus isolated from Salvadora persica from Saudi Arabia. Saudi J Biol Sci 26:5106851077

12. Ellis M (1971) Dematiaceous Hyphomycetes. Commonwealth Mycological Inst., Kew, Surry, England

13. El-Maghraby O, Soltan O, Mohammed R, Mohammed M (2013) Endophytic fungi of three leguminous plant roots in Egypt J Basic Appl Mycol, Egypt. 4:59-68

14. Gilman J (1957) A manual of soil fungi. lowa State Univ. Press, Ames

15. Golinska P, Wypij M, Agarkar G, Rathod D, Dahm H, Rai M (2015) Endophytic actinobacteria of medicinal plants: diversity and bioactivity. Anton Leeuw Int J G 108:2267-2289

16. Gu W (2009) Bioactive metabolites from Alternaria brassicicola ML-P08, an endophytic fungus residing in Malus halliana. World J Microb Biot 25:91677-91683

17. Hardoim PR, van Overbeek LS, Berg G, Pirtillä A, Compant S, Campisano A et al (2015) The hidden world within plants: ecological and evolutionary considerations for defining functioning of microbial endophytes. Microbiol Mol Biol Rev 79:3293-3320

18. Hassan SE (2017) Plant growth-promoting activities for bacterial and fungal endophytes isolated from medicinal plant of Teucrium polium L. J Adv Res 8:6687-6695

19. Hassanein N, El-Gendy M, Abdelhameed N (2016) Endophytic fungi of some medicinal plants in Egypt Egypt. Acad J Biolog Sci 8:165-178

20. Huang WY, Cai YZ, Hyde KD, Corke H, Sun M (2008) Biodiversity of endophytic fungi associated with 29 traditional Chinese medicinal plants. Fungal Divers 33:61-75

21. Hussain T, Llácer JL, Fernández IS, Munoz A, Martin-Marcos P, Savva CG et al (2014) Structural changes enable start codon recognition by the eukaryotic translation initiation complex. Cell 159:3597-3607

22. Jiang S, Duan J, Tao J, Yan H, Zheng J (2010) Ecological distribution and elicitor activities of endophytic fungi in Changium smyrnioides. Chin Tradit Herb Drugs 1:121-125

23. Lou J, Fu L, Peng Y, Zhou L (2013) Metabolites from Alternaria fungi and their bioactivities. Molecules 18:5891-5935

24. Mani VM, Soundari AP, Karthiyaini D, Preethi K (2015) Bioprospecting Endophytic Fungi and Their Metabolites from Medicinal Tree Aegle marmelos in Western Ghats, India. Mycobiology 43:33033310

25. Norrel S, Messley K (1997) Microbiology laboratory manual principles and applications Upper Saddle. Prentice Hall, River 
26. Palanichamy P, Krishnamoorthy G, Kannan S, Marudhamuthu M (2018) Bioactive potential of secondary metabolites derived from medicinal plant endophytes. Egypt J Basic Appl Sci 5:43034312

27. Pielou E (1966) The measurement of diversity in different types of biological collections. J Theor Biol 13:131-144

28. Raper K, Fennel D (1965) The genus Aspergillus. Williams and Wilkins; Baltimore, USA

29. Raper K, Thom C (1949) A manual of penicillia. Williams and Wilkins; Baltimore, USA

30. Raviraj J (2005) Fungal endophytes in five medicinal plant species from Kudremukh Range, Western Ghats of India. J Basic Microbiol 45:3230-3235

31. Saad M, Ghareeb RY, Saeed A (2019) The potential of endophytic fungi as bio-control agents against the cotton leafworm, Spodoptera littoralis (Boisd.) (Lepidoptera: Noctuidae. Egypt J Biol Pest Control 29:7

32. Selim K, Nagia M, El-Ghwas D (2016) Endophytic fungi are multifunctional biosynthesizers:

Ecological role and chemical diversity. In endophytic fungi: diversity, characterization and biocontrol, 1st ed.; NOVA Science Publishers: Hauppauge, NY, USA, 2016

33. Sutjaritvorakul T, Whalley A, Sihanonth P, Roengsumran S (2011) Antimicrobial activity from endophytic fungi isolated from plant leaves in Dipterocarpous forest at Viengsa district Nan province, Thailand. J Agric Tech 6:2309-2315

34. Tonial F, Gomes RR, Gomes-Figueiredo J, Savi DC, Maia B, Glienke C (2016) Influence of culturing conditions on bioprospecting and antimicrobial potential of endophytic fungi from Schinus terebinthifolious. Curr Microbiol 71:1-11

35. Venieraki A, Dimou M, Katinakis P (2017) Endophytic fungi residing in medicinal plants have the ability to produce the same or similar pharmacologically active secondary metabolites as their hosts. Hell. Plant Prot J 10:51-66

36. Whittaker R (1972) Evolution and measurement of species diversity, 21. International Association for Plant Taxonomy, Taxon, pp 213-251

37. Yao YQ, Lan F, Ming Y, Ji Q, Wei G, Shao R et al (2017) Endophytic Fungi Harbored in the Root of Sophora Tonkinensis Gapnep: Diversity and Biocontrol Potential against Phytopathogens. Wiley Microbiol 6:3e00437

\section{Figures}




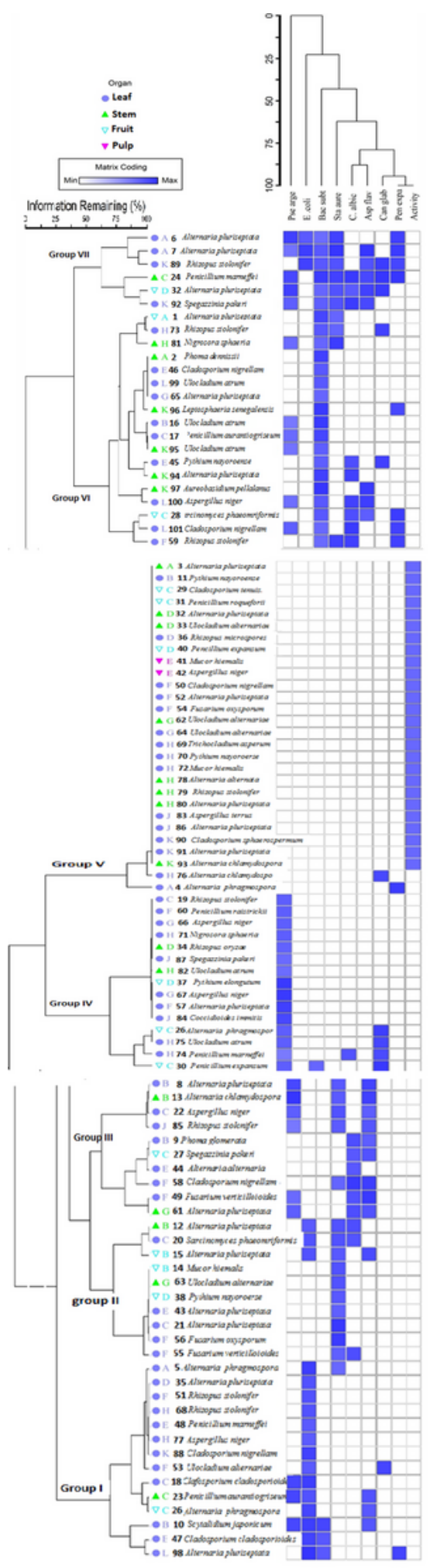

\section{Figure 1}

Two-way cluster analysis dendrogram showing the analysis of the 101isolated endophytes against 8 pathogens. Groups (I - VII) are presented on the hierarchal classification of the isolates. Antimicrobial activity (\%) for each fungi is plotted as a colored squares ranged from the white (no antimicrobial activity) to the blue (the highest activity \%). Intensity of the blue color reflects an isolate activity $\%$ in relation to other isolates. Eleven Plant species are symbolic (A to L). 